\title{
Estimation of the Parameters of the Bivariate Generalized Exponential Distribution using Accelerated Life Testing with Censoring Data
}

\author{
Assar S. M ${ }^{1}$, Abd El-Maseh M. P ${ }^{2 *}$ \\ ${ }^{1}$ Lecturer, Department of Mathematical Statistics Institute of Statistical Studies and Research, Cairo University, Egypt \\ ${ }^{2}$ Master Student, Department of Mathematical Statistics Institute of Statistical Studies and Research, Cairo University, Egypt \\ *Corresponding author E-mail: mary_statistics@yahoo.com
}

Copyright $\odot 2014$ Assar, S. M., Abd El-Maseh, M. P This is an open access article distributed under the Creative Commons Attribution License, which permits unrestricted use, distribution, and reproduction in any medium, provided the original work is properly cited.

\begin{abstract}
In this paper, the estimation for the bivariate generalized exponential (BVGE) distribution under type-I censored with constant stress accelerated life testing (CSALT) are discussed. The scale parameter of the lifetime distribution at constant stress levels is assumed to be an inverse power law function of the stress level. The unknown parameters are estimated by the maximum likelihood approach and their approximate variance-covariance matrix is obtained. Then, the numerical studies are introduced to illustrate the approach study using samples which have been generated from the bivariate generalized exponential distribution.
\end{abstract}

Keywords: Accelerated life testing, Bivariate generalized exponential distribution, Constant stress, Type-I censoring, Maximum likelihood estimation.

\section{Introduction}

In many situations such as the case of the development of a new component or a product failure data at normal operating conditions are lacking and the reliability measure become difficult, if not impossible, to estimate. Indeed, there are cases where the reliability of component is high and failure data of the component when operating at normal conditions (design conditions) may not be attainable during its expected life. In such cases, accelerated life testing (ALT) induces failures, and the failure data at accelerated conditions are used to estimate the reliability at normal operating conditions. Accelerated life testing is usually conducted by subjecting the product (or component) to severer conditions than those that the product will be experiencing at normal conditions or by using the product more intensively than in normal use without changing the normal operating conditions. It is referred to these approaches as accelerated stress and accelerated failure time, respectively. (See Elsayed [7]).

The most common stress loading is constant stress. In constant stress accelerated life test, the stress is kept at a constant level of stress throughout the life of the test; that is, each unit is run at a constant high stress level until the occurrence of failure or the observation is censored. Practically, most devices such as lamps, semiconductors, and microelectronics are run at a constant stress, see Nelson [16]. Many authors have studied statistical inference of CSALT; for example, Lawless [13], AbdelGhaly et al. [1] and Attia et al. [5].

Many times the lifetime data of interest is bivariate in nature. Any Study on twins or on failure data recoded twice on the same system naturally lead to bivariate data. Paired data could also consist of blindness in the left / right eye, failure time of the left /right Kidney or age at death of parent /child in a genetic study. Kundu and Gupta [10] defined a bivariate generalized exponential distribution and provided that it's marginal distributions are generalized exponential (GE) distributions. The joint cumulative distribution function, the joint probability density function and the joint survival distribution function are in closed forms, which make it convenient to use in practice. They used the method of maximum likelihood to estimate the parameters of the BVGE distribution from complete samples. Many authors presented bivariate distribution, Houggard et al. [9] studied data on life length of Danish twins and Lin et al. [14] considered data of colon cancer and the time from treatment to death. Marshal and Olkin [15] considered a bivariate exponential distribution for the life length of two dependence components. Kundu and Gupta [10] analyzed one data set 
and observed that the proposed BVGE distribution provided a much better fit than the Marshal and Olkin bivariate exponential model.

Many authors have studied the properties of the BVGE distribution, for example, Ashour et al. [3] provided the joint and marginal moments of the BVGE distribution, also the joint and marginal moment generating function for the BVGE distribution. Kundu and Gupta [11] introduced an absolute continuous bivariate generalized exponential distribution by using a simple transformation from a well-known bivariate exchangeable distribution. Ashour et al. [4] estimated the unknown parameters of the BVGE distribution from censored type-I samples with random censor time using the method of maximum likelihood. Shoaee and Khorram [17] introduced absolutely continuous baivariate generalized exponential distribution. Attia et al. [6] presented the maximum likelihood estimators for the unknown parameters of bivariate generalized linear failure rate distribution and their approximate variance-covariance matrix.

This paper is organized as follows: The underling BVGE distribution and the assumptions for CSALT for this model are described in Section 2. Section 3 introduces the maximum likelihood estimators (MLEs) of the model parameters, Fisher information matrix and variance-covariance matrix under type-I censoring. The simulation study needed for illustrating the theoretical results are presented in Section 4. Section 5 contains some concluding remarks. Finally, Section 6 contains Acknowledgements for my great people who have assisted me on this paper.

\section{The model}

\subsection{The bivariate generalized exponential distribution}

The CDF for BVGE distribution with the shape parameters $\alpha_{1}, \alpha_{2}, \alpha_{3}>0$ and scale parameter $\lambda>0$ provided as following:

$$
F(x, y)= \begin{cases}F_{1}(x, y) & \text { if } 0<x<y<\infty, \\ F_{2}(x, y) & \text { if } 0<y<x<\infty, \\ F_{3}(x) & \text { if } 0<x=y<\infty,\end{cases}
$$

Where

$$
\begin{aligned}
F_{1}(x, y) & =F_{G E}\left(x ; \alpha_{1}+\alpha_{3}, \lambda\right) F_{G E}\left(y ; \alpha_{2}, \lambda\right) \\
& =\left(1-e^{-\lambda x}\right)^{\alpha_{1}+\alpha_{3}}\left(1-e^{-\lambda y}\right)^{\alpha_{2}} \\
F_{2}(x, y) & =F_{G E}\left(x ; \alpha_{1}, \lambda\right) F_{G E}\left(y ; \alpha_{2}+\alpha_{3}, \lambda\right) \\
& =\left(1-e^{-\lambda x}\right)^{\alpha_{1}}\left(1-e^{-\lambda y}\right)^{\alpha_{2}+\alpha_{3}},
\end{aligned}
$$

and

$$
\begin{aligned}
F_{3}(x) & =f_{G E}\left(x ; \alpha_{1}+\alpha_{2}+\alpha_{3}, \lambda\right) \\
& =\left(1-e^{-\lambda x}\right)^{\alpha_{1}+\alpha_{2}+\alpha_{3}} .
\end{aligned}
$$

where

$F_{G E}(x ; \alpha)=\left(1-e^{-\lambda x}\right)^{\alpha}$.

The PDF for BVGE distribution with the shape parameters $\alpha_{1}, \alpha_{2}, \alpha_{3}>0$ and scale parameter $\lambda>0$ provided as following:

$$
f(x, y)= \begin{cases}f_{1}(x, y) & \text { if } 0<x<y<\infty, \\ f_{2}(x, y) & \text { if } 0<y<x<\infty \\ f_{3}(x) & \text { if } 0<x=y<\infty,\end{cases}
$$

where

$$
\begin{aligned}
f_{1}(x, y) & =f_{G E}\left(x ; \alpha_{1}+\alpha_{3}, \lambda\right) f_{G E}\left(y ; \alpha_{2}, \lambda\right) \\
& =\left(\alpha_{1}+\alpha_{3}\right) \alpha_{2} \lambda^{2}\left(1-e^{-\lambda x}\right)^{\alpha_{1}+\alpha_{3}-1}\left(1-e^{-\lambda y}\right)^{\alpha_{2}-1} e^{-\lambda(x+y)} \\
f_{2}(x, y) & =f_{G E}\left(x ; \alpha_{1}, \lambda\right) f_{G E}\left(y ; \alpha_{2}+\alpha_{3}, \lambda\right) \\
& =\left(\alpha_{2}+\alpha_{3}\right) \alpha_{1} \lambda^{2}\left(1-e^{-\lambda x}\right)^{\alpha_{1}-1}\left(1-e^{-\lambda y}\right)^{\alpha_{2}+\alpha_{3}-1} e^{-\lambda(x+y)}
\end{aligned}
$$

and

$$
\begin{aligned}
f_{3}(x) & =\frac{\alpha_{3}}{\alpha_{1}+\alpha_{2}+\alpha_{3}} f_{G E}\left(x ; \alpha_{1}+\alpha_{2}+\alpha_{3}, \lambda\right) \\
& =\alpha_{3} \lambda\left(1-e^{-\lambda x}\right)^{\alpha_{1}+\alpha_{2}+\alpha_{3}-1} .
\end{aligned}
$$

where

$f_{G E}(x ; \alpha)=\alpha \lambda e^{-\lambda x}\left(1-e^{-\lambda x}\right)^{\alpha-1}$ and The BVGE distribution will be denoted by BVGE $\left(\alpha_{1}, \alpha_{2}, \alpha_{3}, \lambda\right)$.

Note that: Kundu and Gupta [10] provided a special case of the PDF for BVGE distribution with $\lambda=1$ and denoted that the results are true for general $\lambda$ also.

\subsection{Model assumptions}

The assumptions of accelerated life test are assumed to be as following:

i) There are $\mathrm{k}$ levels of high stress $V_{j}, j=1, \ldots, k$ in the experiment and $V_{u}$ is the stress under usual conditions, where $V_{u}<V_{1}<\cdots<V_{k}$. 
ii) A total of $N$ bivariate observations divided into $n_{j}$ bivariate observations for each level of stress $V_{j}$, $j=$ $1,2, \cdots, k$ and $\sum_{j=1}^{k} n_{j}=N$.

iii) For each level of stress the $n_{j}$ bivariate observations divided into $m_{l j}$ bivariate observations where $l=1,2, \ldots, 6$, $j=1,2, \ldots, k$ and $\sum_{l=1}^{6} \sum_{j=1}^{k} m_{l j}=N$.

iv) Each $n_{j}$ bivariate unit, $j=1,2, \ldots, k$ in the experiment is run at a pre-specified constant stress $V_{j}$.

v) It is assumed that the stress $V_{j}, j=1,2, \ldots, k$ affects only the scale parameter $\lambda_{j}$ of the bivariate generalized exponential distribution through a certain acceleration function.

vi) By using the $\operatorname{PDF}(2.1)$ for general scale parameter $\lambda_{j}, j=1,2, \ldots, k$ then the $\left(X_{i j}, Y_{i j}\right)$ observations, $i=$ $1,2, \ldots, n_{j}$ and $j=1,2, \ldots, k$ at stress levels $V_{j}$ are the BVGE distribution function has the joint PDF:

$$
f\left(x_{i j}, y_{i j}\right)= \begin{cases}f_{1}\left(x_{i j}, y_{i j}\right) & \text { if } 0<x_{i j}<y_{i j}<\infty, \\ f_{2}\left(x_{i j}, y_{i j}\right) & \text { if } 0<y_{i j}<x_{i j}<\infty, \\ f_{3}\left(x_{i j}\right) & \text { if } 0<x_{i j}=y_{i j}<\infty,\end{cases}
$$

where

$$
\begin{aligned}
f_{1}\left(x_{i j}, y_{i j}\right) & =f_{G E}\left(x_{i j} ; \alpha_{1}+\alpha_{3}, \lambda_{j}\right) f_{G E}\left(y_{i j} ; \alpha_{2}, \lambda_{j}\right) \\
& =\left(\alpha_{1}+\alpha_{3}\right) \alpha_{2} \lambda_{j}^{2}\left(1-e^{-\lambda_{j} x_{i j}}\right)^{\alpha_{1}+\alpha_{3}-1}\left(1-e^{\left.-\lambda_{j} y_{i j}\right)^{\alpha_{2}-1}} e^{-\lambda_{j}\left(x_{i j}+y_{i j}\right)},\right. \\
f_{2}\left(x_{i j}, y_{i j}\right) & =f_{G E}\left(x_{i j} ; \alpha_{1}, \lambda_{j}\right) f_{G E}\left(y_{i j} ; \alpha_{2}+\alpha_{3}, \lambda_{j}\right) \\
& =\left(\alpha_{2}+\alpha_{3}\right) \alpha_{1} \lambda_{j}^{2}\left(1-e^{-\lambda_{j} x_{i j}}\right)^{\alpha_{1}-1}\left(1-e^{-\lambda_{j} y_{i j}}\right)^{\alpha_{2}+\alpha_{3}-1} e^{-\lambda_{j}\left(x_{i j}+y_{i j}\right)},
\end{aligned}
$$

and

$$
\begin{aligned}
f_{3}\left(x_{i j}\right) & =\frac{\alpha_{3}}{\alpha_{1}+\alpha_{2}+\alpha_{3}} f_{G E}\left(x_{i j} ; \alpha_{1}+\alpha_{2}+\alpha_{3}, \lambda_{j}\right) \\
& =\alpha_{3} \lambda_{j}\left(1-e^{-\lambda_{j} x_{i j}}\right)^{\alpha_{1}+\alpha_{2}+\alpha_{3}-1} .
\end{aligned}
$$

vii) The scale parameter $\lambda_{j}, j=1,2, \ldots, k$ of the underlying life time distribution (2.2) is assumed to have an inverse power law function on stress levels, that is: $\lambda_{j}=C S_{j}^{P}$,

where

$S_{j}=\frac{V^{*}}{V_{j}}, \quad V^{*}=\prod_{j=1}^{k} V_{j}^{b_{j}}, \quad b_{j}=\frac{n_{j}}{N}$,

And $C>0$ is the constant of proportionality and $P>0$ is the power of the applied stress, See, Singpurwalla [20], Abdel-Ghaly et al. [1] and Attia et al. [5].

\section{Maximum likelihood estimation of the parameters}

Suppose that the $N$ bivariate observations under study and $i$-th pair of the components with life-time $\left(x_{i j}, y_{i j}\right)$ have a censoring time $t_{j}$ and the experiment is terminated at a pre-specified censoring time $t_{j}, j=1,2, \ldots, k$.

The life times associated with $i$-th pair of the components is given by

$$
\left(x_{i j}, y_{i j}\right)=\left\{\begin{array}{lr}
\left(x_{i j}, y_{i j}\right) & , \max \left(x_{i j}, y_{i j}\right)<t_{j}, \\
\left(x_{i j}, t_{j}\right) & , x_{i j}<t_{j}<y_{i j}, \\
\left(t_{j}, y_{i j}\right) & , y_{i j}<t_{j}<x_{i j}, \\
\left(t_{j}, t_{j}\right) & , t_{j}<\min \left(x_{i j}, y_{i j}\right) .
\end{array}\right.
$$

According to Hanagal [8], the likelihood function under type-I censoring with CSALT of the sample size $N$ bivariate observations is given by:

$$
\begin{aligned}
L\left(\alpha_{1}, \alpha_{2}, \alpha_{3}, C, P\right)= & \prod_{j=1}^{k}\left\{\left[\prod_{i=1}^{m_{1 j}} f_{A_{1}}\left(x_{i j}, y_{i j}\right) \overline{G_{A}}\left(t_{j}\right)\right]\left[\prod_{i=1}^{m_{2 j}} f_{A_{2}}\left(x_{i j}, y_{i j}\right) \overline{G_{A}}\left(t_{j}\right)\right]\left[\prod_{i=1}^{m_{3 j}} f_{A_{3}}\left(x_{i j}, y_{i j}\right) \overline{G_{A}}\left(t_{j}\right)\right]\right. \\
& \left.\cdot\left[\prod_{i=1}^{m_{4 j}} f_{A_{4}}\left(x_{i j}, t_{j}\right) g_{A}\left(t_{j}\right)\right]\left[\prod_{i=1}^{m_{5 j}} f_{A_{5}}\left(t_{j}, y_{i j}\right) g_{A}\left(t_{j}\right)\right]\left[m_{6 j} \overline{F_{A}}\left(t_{j}, t_{j}\right) g_{A}\left(t_{j}\right)\right]\right\} .
\end{aligned}
$$

where: $f_{A}\left(x_{i j}, y_{i j}\right)$ and $g_{A}\left(t_{j}\right)$ are the joint PDF with accelerated life testing of $\left(X_{i j}, Y_{i j}\right)$ and $T_{j}$ respectively, $\overline{F_{A}}\left(t_{j}, t_{j}\right)$ and $\overline{G_{A}}\left(t_{j}\right)$ are survivor function with life testing of $\left(T_{j}, T_{j}\right)$ and $T_{j}$ respectively,

$$
\begin{aligned}
g_{A}\left(t_{j}\right) & =C S_{j}^{P} e^{-C S_{j}^{P} t_{j}} \quad ; t_{j}>0, \quad C, P>0 . \\
\overline{G_{A}}\left(t_{j}\right) & =P\left[T_{j}>\max \left(x_{i j}, y_{i j}\right)\right] \\
& =\exp \left[-C S_{j}^{P} \max \left(x_{i j}, y_{i j}\right)\right], \\
f_{A_{1}}\left(x_{i j}, y_{i j}\right) & =f_{G E}\left(x_{i j} ; \alpha_{1}+\alpha_{3}, C, P\right) f_{G E}\left(y_{i j} ; \alpha_{2}, C, P\right) \\
& =\left(\alpha_{1}+\alpha_{3}\right) \alpha_{2} C^{2} S_{j}^{2 P}\left(1-e^{-C S_{j}^{P} x_{i j}}\right)^{\alpha_{1}+\alpha_{3}-1}\left(1-e^{-C S_{j}^{P} y_{i j}}\right)^{\alpha_{2}-1} e^{-C S_{j}^{P}\left(x_{i j}+y_{i j}\right)}, \\
f_{A_{2}}\left(x_{i j}, y_{i j}\right) & =f_{G E}\left(x_{i j} ; \alpha_{1}, C, P\right) f_{G E}\left(y_{i j} ; \alpha_{2}+\alpha_{3}, C, P\right)
\end{aligned}
$$




$$
\begin{aligned}
f_{A_{2}}\left(x_{i j}, y_{i j}\right) & =\left(\alpha_{2}+\alpha_{3}\right) \alpha_{1} C^{2} S_{j}^{2 P}\left(1-e^{-C S_{j}^{P} x_{i j}}\right)^{\alpha_{1}-1}\left(1-e^{-C S_{j}^{P} y_{i j}}\right)^{\alpha_{2}+\alpha_{3}-1} e^{-C S_{j}^{P}\left(x_{i j}+y_{i j}\right)}, \\
f_{A_{3}}\left(x_{i j}\right) & =\frac{\alpha_{3}}{\alpha_{1}+\alpha_{2}+\alpha_{3}} f_{G E}\left(x_{i j} ; \alpha_{1}+\alpha_{2}+\alpha_{3}, C, P\right) \\
& =\alpha_{3} C S_{j}^{P}\left(1-e^{-C S_{j}^{P} x_{i j}}\right)^{\alpha_{1}+\alpha_{2}+\alpha_{3}-1}, \\
f_{A_{4}}\left(x_{i j}, t_{j}\right) & =\lim _{\delta x_{i j} \rightarrow 0} \frac{P\left(x_{i j}<x_{i j}<x_{i j}+\delta x_{i j} \mid Y_{i j}>t_{j}\right) P\left(Y_{i j}>t_{j}\right)}{\delta x_{i j}} \\
& =C S_{j}^{P}\left(\alpha_{1}+\alpha_{3}\right) e^{-C S_{j}^{P} x_{i j}}\left(1-e^{-C S_{j}^{P} x_{i j}}\right)^{\alpha_{1}+\alpha_{3}-1}\left[1-\left(1-e^{-C S_{j}^{P} t_{j}}\right)^{\alpha_{2}}\right],
\end{aligned}
$$

where $0<x_{i j}<t_{j}, C>0$ and $P>0$.

$$
\begin{aligned}
f_{A_{5}}\left(t_{j}, y_{i j}\right) & =\lim _{\delta y_{i j} \rightarrow 0} \frac{P\left(y_{i j}<Y_{i j}<y_{i j}+\delta y_{i j} \mid x_{i j}>t_{j}\right) P\left(x_{i j}>t_{j}\right)}{\delta y_{i j}} \\
& =C S_{j}^{P}\left(\alpha_{2}+\alpha_{3}\right) e^{-C S_{j}^{P} y_{i j}}\left(1-e^{-C S_{j}^{P} y_{i j}}\right)^{\alpha_{2}+\alpha_{3}-1}\left[1-\left(1-e^{-C S_{j}^{P} t_{j}}\right)^{\alpha_{1}}\right],
\end{aligned}
$$

where $0<y_{i j}<t_{j}, C>0$ and $P>0$.

$$
\text { and } \begin{aligned}
\bar{F}\left(t_{j}, t_{j}\right) & =P\left[X_{i j}>t_{j}, Y_{i \mathrm{j}}>t_{j}\right] \\
& =1-\left(1-e^{-C S_{j}^{P} t_{j}}\right)^{\alpha_{1}+\alpha_{2}+\alpha_{3}} .
\end{aligned}
$$

Assumed that $C$ and $\alpha_{3}$ are known, the likelihood function (3.1) reduced to:

$$
\begin{aligned}
L\left(x, y \mid \alpha_{1}, \alpha_{2}, P\right)= & B \alpha_{1}^{\vartheta_{2}} \alpha_{2}^{\vartheta_{1}}\left(\alpha_{1}+\alpha_{3}\right)^{\vartheta_{3}}\left(\alpha_{2}+\alpha_{3}\right)^{\vartheta_{4}} \cdot \prod_{j=1}^{k}\left\{m_{6 j} S_{j}^{\left(U_{1 j}+U_{2 j}\right) P} e^{-C S_{j}^{P} U_{3 j}}\left[1-\Delta\left(t_{j}\right)^{\alpha_{1}+\alpha_{2}+\alpha_{3}}\right]\right. \\
& \cdot \prod_{i=1}^{m_{1 j}}\left[\Delta\left(x_{i j}\right)^{\alpha_{1}+\alpha_{3}-1} \Delta\left(y_{i j}\right)^{\alpha_{2}-1}\right] \prod_{i=1}^{m_{2 j}}\left[\Delta\left(x_{i j}\right)^{\alpha_{1}-1} \Delta\left(y_{i j}\right)^{\alpha_{2}+\alpha_{3}-1}\right] \prod_{i=1}^{m_{3 j}}\left[\Delta\left(x_{i j}\right)^{\alpha_{1}+\alpha_{2}+\alpha_{3}-1}\right] \\
& \left.\cdot \prod_{i=1}^{m_{4 j}}\left[\Delta\left(x_{i j}\right)^{\alpha_{1}+\alpha_{3}-1}\left(1-\Delta\left(t_{j}\right)^{\alpha_{2}}\right)\right] \prod_{i=1}^{m_{5 j}}\left[\Delta\left(y_{i j}\right)^{\alpha_{2}+\alpha_{3}-1}\left(1-\Delta\left(t_{j}\right)^{\alpha_{1}}\right)\right]\right\} .
\end{aligned}
$$

where $B$ is the constant of proportionality,

$$
\begin{array}{rlr}
\Delta\left(x_{i j}\right) & =1-e^{-c S_{j}^{P} x_{i j},} & \text { for all } i=1,2, \ldots, n_{j} \text { and } j=1,2, \ldots, k, \\
\vartheta_{3} & =\sum_{j=1}^{k}\left(m_{1 j}+m_{4 j}\right), & \text { for all } j=1,2, \ldots, k \text { and } r=1,2, \\
\vartheta_{4} & =\sum_{j=1}^{k}\left(m_{2 j}+m_{5 j}\right), & \text { for all } j=1,2, \ldots, k, \\
U_{1 j} & =2\left(m_{1 j}+m_{2 j}+m_{4 j}+m_{5 j}\right), & \text { for all } j=1,2, \ldots, k, \\
U_{2 j} & =m_{3 j}+m_{6 j}, & \text { for all } j=1,2, \ldots, k, \\
\mathrm{U}_{3 \mathrm{j}} & =\sum_{\mathrm{i}=1}^{\mathrm{m}_{1 j}}\left[\left(\mathrm{x}_{\mathrm{ij}}+\mathrm{y}_{\mathrm{ij}}\right)+\max _{2}\left(\mathrm{x}_{\mathrm{ij}}, \mathrm{y}_{\mathrm{ij}}\right)\right]+\sum_{\mathrm{i}=1}^{\mathrm{m}_{2 \mathrm{j}}}\left[\left(\mathrm{x}_{\mathrm{ij}}+\mathrm{y}_{\mathrm{ij}}\right)+\max \left(\mathrm{x}_{\mathrm{ij}}, \mathrm{y}_{\mathrm{ij}}\right)\right]+\sum_{\mathrm{i}=1}^{\mathrm{m}_{3 \mathrm{j}}}\left[\max \left(\mathrm{x}_{\mathrm{ij}}, \mathrm{y}_{\mathrm{ij}}\right)\right] \\
& +\sum_{\mathrm{i}=1}^{\mathrm{m}_{4 j}}\left(\mathrm{x}_{\mathrm{ij}}+\mathrm{t}_{\mathrm{j}}\right)+\sum_{\mathrm{i}=1}^{\mathrm{m}_{5 j}}\left(\mathrm{y}_{\mathrm{ij}}+\mathrm{t}_{\mathrm{j}}\right)+\left(\mathrm{m}_{6 \mathrm{j}} \mathrm{t}_{\mathrm{j}}\right), & \text { for all } \mathrm{i}=1,2, \ldots, \mathrm{n}_{\mathrm{j}} \text { and } \mathrm{j}=1,2, \ldots, \mathrm{k} .
\end{array}
$$

The log-likelihood function for Equation (3.2) will be as:

$\ln \left[L\left(\alpha_{1}, \alpha_{2}, P\right)\right]=\ln (B)+\vartheta_{2} \ln \left(\alpha_{1}\right)+\vartheta_{1} \ln \left(\alpha_{2}\right)+\vartheta_{3} \ln \left(\alpha_{1}+\alpha_{3}\right)+\vartheta_{4} \ln \left(\alpha_{2}+\alpha_{3}\right)+\sum_{j=1}^{k}\left\{P \ln \left(S_{j}\right)\left(U_{1 j}+U_{2 j}\right)\right.$

$$
\begin{aligned}
& -C S_{j}^{P} U_{3 j}+\left(\alpha_{1}+\alpha_{3}-1\right)\left[\sum_{i=1}^{m_{1 j}}\left(L\left(x_{i j}\right)\right)+\sum_{i=1}^{m_{4 j}}\left(L\left(x_{i j}\right)\right)\right]+\left(\alpha_{2}-1\right) \sum_{i=1}^{m_{1 j}}\left(L\left(y_{i j}\right)\right)+\left(\alpha_{1}-1\right) \\
\cdot & \sum_{i=1}^{m_{2 j}}\left(L\left(x_{i j}\right)\right)+\left(\alpha_{2}+\alpha_{3}-1\right)\left[\sum_{i=1}^{m_{2 j}}\left(L\left(y_{i j}\right)\right)+\sum_{i=1}^{m_{5 j}}\left(L\left(y_{i j}\right)\right)\right]+\left(\alpha_{1}+\alpha_{2}+\alpha_{3}-1\right) \\
\cdot & \left.\sum_{i=1}^{m_{3 j}}\left(L\left(x_{i j}\right)\right)+m_{4 j} W_{2 j}+m_{5 j} W_{1 j}+m_{6 j} W_{3 j}\right\}
\end{aligned}
$$

where

$$
\begin{aligned}
L\left(x_{i j}\right) & =\ln \left(1-\Delta\left(x_{i j}\right)\right), \\
W_{r j} & =\ln \left[1-\left(1-\Delta\left(t_{j}\right)\right)^{\alpha_{r}}\right], \\
W_{3 j} & =\ln \left[1-\Delta\left(t_{j}\right)^{\alpha_{1}+\alpha_{2}+\alpha_{3}}\right],
\end{aligned}
$$

and

for all $i=1,2, \ldots, n_{j}$ and $j=1,2, \ldots, k$, for all $j=1,2, \ldots, k$ and $r=1,2$,

for all $j=1,2, \ldots, k$,

The first partial derivatives of the log-likelihood function (3.3) with respect to the unknown parameters $P, \alpha_{1}$ and $\alpha_{2}$ are as following:

$$
\begin{aligned}
\frac{\partial \ln (L)}{\partial P} & =\sum_{j=1}^{k}\left\{\left[\left(U_{1 j}+U_{2 j}\right) \ln \left(S_{j}\right)\right]-U_{4 j} U_{3 j} \ln \left(S_{j}\right)+\left(\alpha_{1}+\alpha_{3}-1\right) \sum_{i=1}^{m_{1 j}}\left(\psi\left(x_{i j}\right)\right)+\left(\alpha_{2}-1\right) \sum_{i=1}^{m_{1 j}}\left(\psi\left(y_{i j}\right)\right)\right. \\
& +\left(\alpha_{1}-1\right) \sum_{i=1}^{m_{2 j}}\left(\psi\left(x_{i j}\right)\right)+\left(\alpha_{2}+\alpha_{3}-1\right)\left[\sum_{i=1}^{m_{2 j}}\left(\psi\left(y_{i j}\right)\right)+\sum_{i=1}^{m_{5 j}}\left(\psi\left(y_{i j}\right)\right)\right]+\left(\alpha_{1}+\alpha_{2}+\alpha_{3}-1\right) \sum_{i=1}^{m_{3 j}}\left(\psi\left(x_{i j}\right)\right) \\
& \left.+\left(\alpha_{1}+\alpha_{3}-1\right) \sum_{i=1}^{m_{4 j}}\left(\psi\left(x_{i j}\right)\right)+m_{4 j} \varphi_{2 j}+m_{5 j} \varphi_{1 j}+m_{6 j} \varphi_{3 j}\right\}
\end{aligned}
$$

where

$$
\begin{aligned}
U_{4 j} & =C S_{j}^{P}, \\
\psi\left(x_{i j}\right) & =\frac{U_{4 j} x_{i j} \ln \left(s_{j}\right) e^{-U_{4 j} x_{i j}}}{1-e^{-U_{4 j} x_{i j}}}, \\
\varphi_{r j} & =\frac{\alpha_{r} U_{4 j} t_{j} \ln \left(s_{j}\right) e^{-U_{4 j} t_{j}}\left(t_{j}\right)^{\alpha_{r}-1}}{1-\Delta\left(t_{j}\right)^{\alpha_{r}}},
\end{aligned}
$$

for all $j=1,2, \ldots, k$,

and

$$
\varphi_{3 j}=\frac{\left(\alpha_{1}+\alpha_{2}+\alpha_{3}\right) U_{4 j} t_{j} \ln \left(S_{j}\right) e^{-U_{4 j} t_{j}} \Delta\left(t_{j}\right)^{\alpha_{1}+\alpha_{2}+\alpha_{3}-1}}{1-\Delta\left(t_{j}\right)^{\alpha_{1}+\alpha_{2}+\alpha_{3}}},
$$




$$
\begin{aligned}
\frac{\partial \ln (L)}{\partial \alpha_{1}} & =\frac{\vartheta_{2}}{\alpha_{1}}+\frac{\vartheta_{3}}{\alpha_{1}+\alpha_{3}}+\sum_{j=1}^{k}\left\{\sum_{i=1}^{m_{1 j}} L\left(x_{i j}\right)+\sum_{i=1}^{m_{2 j}} L\left(x_{i j}\right)+\sum_{i=1}^{m_{3 j}} L\left(x_{i j}\right)+\sum_{i=1}^{m_{4 j}} L\left(x_{i j}\right)-\frac{m_{5 j} \Delta\left(t_{j}\right)^{\alpha_{1}} L\left(t_{j}\right)}{1-\Delta\left(t_{j}\right)^{\alpha_{1}}}\right. \\
& \left.-\frac{m_{6 j} \Delta\left(t_{j}\right)^{\alpha_{1}+\alpha_{2}+\alpha_{3}} L\left(t_{j}\right)}{1-\Delta\left(t_{j}\right)^{\alpha_{1}+\alpha_{2}+\alpha_{3}}}\right\},
\end{aligned}
$$

and

$$
\begin{aligned}
\frac{\partial \ln (L)}{\partial \alpha_{2}} & =\frac{\vartheta_{1}}{\alpha_{2}}+\frac{\vartheta_{4}}{\alpha_{2}+\alpha_{3}}+\sum_{j=1}^{k}\left\{\sum_{i=1}^{m_{1 j}} L\left(y_{i j}\right)+\sum_{i=1}^{m_{2 j}} L\left(y_{i j}\right)+\sum_{i=1}^{m_{3 j}} L\left(x_{i j}\right)+\sum_{i=1}^{m_{5 j}} L\left(y_{i j}\right)-\frac{m_{4 j} \Delta\left(t_{j}\right)^{\alpha_{2}} L\left(t_{j}\right)}{1-\Delta\left(t_{j}\right)^{\alpha_{2}}}\right. \\
& -\frac{m_{6 j} \Delta\left(t_{j}\right)^{\alpha_{1}+\alpha_{2}+\alpha_{3}} L\left(t_{j}\right)}{1-\Delta\left(t_{j}\right)^{\alpha_{1}+\alpha_{2}+\alpha_{3}}}
\end{aligned}
$$

The solutions for the first partial derivatives equations (3.4), (3.5) and (3.6) cannot be obtained theoretically. So that, a numerical technique such as Mathcad Package will be used to obtain the solutions numerically.

The elements of the Fisher information matrix $(F)$ for the MLEs can be obtained as a symmetric matrix of negative second partial derivatives and the derivatives evaluated using the MLEs $\hat{P}, \hat{\alpha}_{1}$ and $\hat{\alpha}_{2}$, The asymptotic variancecovariance matrix $(\Sigma)$ for the MLEs is defined as the inverse of Fisher information matrix that is,

$$
F=-\left[\begin{array}{ccc}
\frac{\partial^{2} \ln (L)}{\partial \hat{P}^{2}} & \frac{\partial^{2} \ln (L)}{\partial \hat{P} \partial \widehat{\alpha}_{1}} & \frac{\partial^{2} \ln (L)}{\partial \widehat{P} \partial \widehat{\alpha}_{2}} \\
\frac{\partial^{2} \ln (L)}{\partial \widehat{\alpha}_{1} \partial \hat{P}} & \frac{\partial^{2} \ln (L)}{\partial \widehat{\alpha}_{1}^{2}} & \frac{\partial^{2} \ln (L)}{\partial \widehat{\alpha}_{1} \partial \widehat{\alpha}_{2}} \\
\frac{\partial^{2} \ln (L)}{\partial \widehat{\alpha}_{2} \partial \hat{P}} & \frac{\partial^{2} \ln (L)}{\partial \widehat{\alpha}_{2} \partial \widehat{\alpha}_{1}} & \frac{\partial^{2} \ln (L)}{\partial \widehat{\alpha}_{2}^{2}}
\end{array}\right],
$$

where the second partial derivatives of the log-likelihood function (3.3) with respect to the unknown parameters $P, \alpha_{1}$ and $\alpha_{2}$ are as following:

$$
\begin{aligned}
\frac{\partial^{2} \ln (L)}{\partial P^{2}} & =\sum_{j=1}^{k}\left\{-\left[U_{4 j} U_{3 j} \ln \left(S_{j}\right)^{2}\right]+\left(\alpha_{1}+\alpha_{3}-1\right) \sum_{i=1}^{m_{1 j}}\left[D\left(x_{i j}\right)\right]+\left(\alpha_{2}-1\right) \sum_{i=1}^{m_{1 j}}\left(D_{y_{i j}}\right)+\left(\alpha_{1}-1\right) \sum_{i=1}^{m_{2 j}}\left[D\left(x_{i j}\right)\right]\right. \\
& +\left(\alpha_{1}+\alpha_{2}+\alpha_{3}-1\right) \sum_{i=1}^{m_{3 j}}\left[D\left(x_{i j}\right)\right]+\left(\alpha_{2}+\alpha_{3}-1\right)\left[\sum_{i=1}^{m_{2 j}}\left[D\left(y_{i j}\right)\right]+\sum_{i=1}^{m_{5 j}}\left[D\left(y_{i j}\right)\right]\right]+\left(\alpha_{1}+\alpha_{3}-1\right) \\
& \left.\cdot \sum_{i=1}^{m_{4 j}}\left[D\left(x_{i j}\right)\right]+m_{4 j} H_{2 j}+m_{5 j} H_{1 j}+m_{6 j} H_{3 j}\right\},
\end{aligned}
$$

where

$$
\begin{aligned}
& D\left(x_{i j}\right)=\frac{U_{4 j} x_{i j}\left[\ln \left(S_{j}\right)\right]^{2} e^{-U_{4 j} x_{i j}\left\{\left(1-U_{4 j} x_{i j}\right) \Delta\left(x_{i j}\right)-U_{4 j} x_{i j} e^{-U_{4 j} x_{i j}}\right\}}}{\Delta\left(x_{i j}\right)^{2}}, \\
& H_{r j}=\frac{\alpha_{r} U_{4 j} t_{j}\left[\ln \left(s_{j}\right)\right]^{2} e^{-2 U_{4 j} t_{j}} \Delta\left(t_{j}\right)^{\alpha_{r}-1}}{\left[1-\Delta\left(t_{j}\right)^{\alpha_{r}}\right]^{2}} \cdot\left\{\left[e^{U_{4 j} t_{j}}-U_{4 j} t_{j} e^{U_{4 j} t_{j}}+\left(\alpha_{1}-1\right) U_{4 j} t_{j}\right]\left[1-\Delta\left(t_{j}\right)^{\alpha_{r}}\right]-\alpha_{1} U_{4 j} t_{j} \Delta\left(t_{j}\right)^{\alpha_{r}-1}\right\}, \\
& \text { for all } r=1,2,
\end{aligned}
$$

$$
\text { and } \begin{aligned}
& H_{3 j}= \frac{\left(\alpha_{1}+\alpha_{2}+\alpha_{3}\right) U_{4 j} t_{j}\left[\ln \left(s_{j}\right)\right]^{2} e^{-2 U_{4 j} t_{j}} \Delta\left(t_{j}\right)^{\alpha_{1}+\alpha_{2}+\alpha_{3}-1}}{\left[1-\Delta\left(t_{j}\right)^{\alpha_{1}+\alpha_{2}+\alpha_{3}}\right]^{2}} \cdot\left\{\left[e^{U_{4 j} t_{j}}-U_{4 j} t_{j} e^{U_{4 j} t_{j}}+\left(\alpha_{1}+\alpha_{2}+\alpha_{3}-1\right) U_{4 j} t_{j}\right]\right. \\
&\left.\cdot\left[1-\Delta\left(t_{j}\right)^{\alpha_{1}+\alpha_{2}+\alpha_{3}}\right]-\left(\alpha_{1}+\alpha_{2}+\alpha_{3}\right) U_{4 j} t_{j} \Delta\left(t_{j}\right)^{\alpha_{1}+\alpha_{2}+\alpha_{3}-1}\right\}, \\
& \frac{\partial^{2} \ln (L)}{\partial \alpha_{1}^{2}}=-\frac{\vartheta_{2}}{\alpha_{1}^{2}}-\frac{\vartheta_{3}}{\left(\alpha_{1}+\alpha_{3}\right)^{2}}-\sum_{j=1}^{k}\left(m_{5 j} Q_{1 j}+m_{6 j} Q_{3 j}\right),
\end{aligned}
$$

where

$$
Q_{r j}=\frac{\Delta\left(t_{j}\right)^{\alpha_{r}} L\left(t_{j}\right)^{2}\left[1-2 \Delta\left(t_{j}\right)^{\alpha_{r}}\right]}{\left[1-\Delta\left(t_{j}\right)^{\alpha_{r}}\right]^{2}}
$$

and

$$
\begin{aligned}
Q_{3 j} & =\frac{\Delta\left(t_{j}\right)^{\alpha_{1}+\alpha_{2}+\alpha_{3}} L\left(t_{j}\right)^{2}\left[1-2 \Delta\left(t_{j}\right)^{\alpha_{1}+\alpha_{2}+\alpha_{3}}\right]}{\left[1-\Delta\left(t_{j}\right)^{\alpha_{1}+\alpha_{2}+\alpha_{3}}\right]^{2}}, \\
\frac{\partial^{2} \ln (L)}{\partial \alpha_{2}^{2}} & =-\frac{\vartheta_{1}}{\alpha_{2}^{2}}-\frac{\vartheta_{4}}{\left(\alpha_{2}+\alpha_{3}\right)^{2}}-\sum_{j=1}^{k}\left(m_{4 j} Q_{2 j}+m_{6 j} Q_{3 j}\right), \\
\frac{\partial^{2} \ln (L)}{\partial \alpha_{1} \partial P} & =\sum_{j=1}^{k}\left\{\sum_{i=1}^{m_{1 j}} \psi\left(x_{i j}\right)+\sum_{i=1}^{m_{2 j}} \psi\left(x_{i j}\right)+\sum_{i=1}^{m_{3 j}} \psi\left(x_{i j}\right)+\sum_{i=1}^{m_{4 j}} \psi\left(x_{i j}\right)-m_{5 j} Z_{1 j}-m_{6 j} Z_{3 j}\right\},
\end{aligned}
$$

where

$$
Z_{r j}=\frac{U_{4 j} t_{j} e^{-U_{4 j} t_{j}} \Delta\left(t_{j}\right)^{\alpha_{r}-1} \ln \left(s_{j}\right)}{\left(1-\Delta\left(t_{j}\right)^{\alpha_{r}}\right)^{2}}\left\{\left[1+\alpha_{r} L\left(t_{j}\right)\right]\left[1-\Delta\left(t_{j}\right)^{\alpha_{r}}\right]-\alpha_{1} \Delta\left(t_{j}\right)^{\alpha_{r}} L\left(t_{j}\right)\right\},
$$

and

$$
\begin{aligned}
Z_{3 j}= & \frac{U_{4 j} t_{j} e^{-U_{4 j} t_{j}} \Delta\left(t_{j}\right)^{\alpha_{1}+\alpha_{2}+\alpha_{3}-1} \ln \left(s_{j}\right)}{\left[1-\Delta\left(t_{j}\right)^{\alpha_{1}+\alpha_{2}+\alpha_{3}}\right]^{2}} \cdot\left\{\left[1+\left(\alpha_{1}+\alpha_{2}+\alpha_{3}\right) L\left(t_{j}\right)\right]\left[1-\Delta\left(t_{j}\right)^{\alpha_{1}+\alpha_{2}+\alpha_{3}}\right]-\left(\alpha_{1}+\alpha_{2}+\alpha_{3}\right)\right. \\
& \left.\cdot \Delta\left(t_{j}\right)^{\alpha_{1}+\alpha_{2}+\alpha_{3}} L\left(t_{j}\right)\right\} . \\
\frac{\partial^{2} \ln (L)}{\partial \alpha_{2} \partial P}= & \sum_{j=1}^{k}\left\{\sum_{i=1}^{m_{1 j}} \psi\left(y_{i j}\right)+\sum_{i=1}^{m_{2 j}} \psi\left(y_{i j}\right)+\sum_{i=1}^{m_{3 j}} \psi\left(x_{i j}\right)+\sum_{i=1}^{m_{5 j}} \psi\left(y_{i j}\right)-m_{4 j} Z_{2 j}-m_{6 j} Z_{3 j}\right\},
\end{aligned}
$$


and

$\frac{\partial^{2} \ln (L)}{\partial \alpha_{2} \partial \alpha_{1}}=-\sum_{j=1}^{k}\left(m_{6 j} Q_{3 j}\right)$.

\section{Simulation study}

To illustrate the theoretical results, a numerical example will be given to obtain the MLEs of unknown parameters $P, \alpha_{1}$ and $\alpha_{2}$ and their approximate variance-covariance matrix using Mathcad software. The simulation procedures are described through the following steps:

i) Consider three accelerated stress levels $V_{1}=0.75, V_{2}=1.5, V_{3}=2.25$ and assume that usual stress is $V_{u}=0.5$.

ii) Assume that the experiment is terminated at a specified time $t_{j}, j=1,2,3$, where, $t_{1}=4, t_{2}=3, t_{3}=2$.

iii) Generating random samples under usual stress of size $n_{u}$ bivariate observations from the BVGE distribution with parameter $\lambda=0.25, \alpha_{1}=2, \alpha_{2}=1.5$ and $\alpha_{3}=1$ as following:

- $\quad$ Generate $n_{u}$ bivariate observations from uniform $(0,1)$.

- Generate $\left\{v_{11}, \ldots, v_{1 n_{u}}\right\}$ from $\operatorname{GE}\left(\alpha_{1}, \lambda\right)$, similarly, $\left\{v_{21}, \ldots, v_{2 n_{u}}\right\}$ from $\operatorname{GE}\left(\alpha_{2}, \lambda\right),\left\{v_{31}, \ldots, v_{3 n_{u}}\right\}$ from $\operatorname{GE}\left(\alpha_{3}, \lambda\right)$ Obtain $x_{i 1}=\max \left\{v_{1 i}, v_{3 i}\right\}$ and $y_{i 1}=\max \left\{v_{2 i}, v_{3 i}\right\}$, for $i=1, \ldots, n_{u}$.

Thus, generated random samples for usual stress of size $n_{u}=25,50,75,150$ from a BVGE distribution are presented with $\lambda=0.25, \alpha_{1}=2, \alpha_{2}=1.95$ and $\alpha_{3}=1$, see, kundu and Gupta [12].

iv) The Kolmgrov-Simrnov (K-S) test is used for assessing that the data set follows the BVGE distribution; see AlMutrairi et al. [2].

v) The $m_{1 j}, m_{2 j}, m_{3 j}, m_{4 j}, m_{5 j}$ and $m_{6 j}$ for the generated BVGE random numbers are calculated as following: $m_{1 j}=\sum_{i=1}^{n_{j}} a_{i j}, \quad m_{2 j}=\sum_{i=1}^{n_{j}} c_{i j}, \quad m_{3 j}=\sum_{i=1}^{n_{j}} f_{i j}, \quad m_{4 j}=\sum_{i=1}^{\mathrm{n}_{j}} b_{i j}, \quad m_{5 j}=\sum_{i=1}^{n_{j}} d_{i j}, \quad m_{6 j}=\sum_{i=1}^{n_{j}} p_{i j}$. where

$a_{i j}=\left\{\begin{array}{rr}1 & \text { if } X_{i j}<Y_{i j}<T_{j} \\ 0 & \text { if otherwise }\end{array}\right.$,

$b_{i j}=\left\{\begin{array}{cc}1 & \text { if } X_{i j}<T_{j}<Y_{i j}\end{array}\right.$

$c_{i j}=\left\{\begin{array}{cc}1 & \text { if } Y_{i j}<X_{i j}<T_{j} \\ 0 & \text { if otherwise }\end{array}\right.$

$d_{i j}= \begin{cases}1 & \text { if } Y_{i j}<T_{j}<X_{i j} \\ 0 & \text { if otherwise }\end{cases}$

$f_{i j}=\left\{\begin{array}{ccc}1 & \text { if } & 0<\left(X_{i j}=Y_{i j}\right)<T_{j} \\ 0 & \text { if } & \text { otherwise }\end{array}\right.$,

$p_{i j}=\left\{\begin{array}{ll}1 & \text { if } \min \left(X_{i j}, Y_{i j}\right)>T_{j} \\ 0 & \text { if otherwise }\end{array}\right.$.

vi) At each stress level 25, 50, 75, 150 bivariate observations $(X, Y)$ was generated from a BVGE distribution then $N=75,150,225,450$.

vii) Assume that $C=0.5$ and $\alpha_{3}=1$ and the initial values $P=-0.25, \alpha_{1}=2$ and $\alpha_{2}=1.95$, using the likelihood equation (3.2) the MLEs $\hat{P}, \hat{\alpha}_{1}$ and $\hat{\alpha}_{2}$ will be obtained using iterative procedure using Mathcad Software, their approximate variance-covariance matrix, relative bias (RAB), mean square error (MSE) and a $99.99 \%$ asymptotic confidence intervals are displayed in Table 1.

Table 1: The Estimates, Relative Bais, MSE and Confidence Interval.

\begin{tabular}{lllll}
\hline$N$ & parameter & MLE & MSE & Confidence Intervals \\
\hline \multirow{3}{*}{75} & $P$ & -0.410 & 0.427 & $(-0.822,0.002)$ \\
& $\alpha_{1}$ & 2.761 & 0.518 & $(0.873,4,649)$ \\
& $\alpha_{2}$ & 1.561 & 0.321 & $(-0.076,3.198)$ \\
\hline \multirow{3}{*}{150} & $P$ & -0.411 & 0.424 & $(-0.701,-0.121)$ \\
& $\alpha_{1}$ & 2.650 & 0.272 & $(1.288,3.912)$ \\
& $\alpha_{2}$ & 1.470 & 0.186 & $(0.378,2.562)$ \\
\hline \multirow{3}{*}{25} & $P$ & -0.41 & 0.415 & $(1.616,3.662)$ \\
& $\alpha_{1}$ & 2.639 & 0.212 & $(0.576,2.35)$ \\
\hline \multirow{3}{*}{450} & $\alpha_{2}$ & 1.463 & 0.145 & $(-0.577,-0.243)$ \\
& $P$ & -0.41 & 0.413 & $(1.879,3.291)$ \\
& $\alpha_{1}$ & 2.585 & 0.138 & $(0.803,2.025)$ \\
\hline
\end{tabular}




\section{Conclusion}

This paper presented statistical inference for BVGE distribution under CSALT with k-stress levels. The scale parameter was assumed to be an inverse power law function of the stress level. The MLEs are obtained for the unknown parameter $P, \alpha_{1}$ and $\alpha_{2}$ for BVGE distribution under type-I censored data with CSALT. Then, the MLEs are calculated numerically for the unknown parameters under type-I censoring and CSALT. By using MLEs it is obtained the values for their approximate variance-covariance matrix, relative bias, mean square error and $99.99 \%$ asymptotic confidence intervals. Finally, Mathcad technique is used to obtain the numerical results for the proposed model, then, we conclude that the MSE for unknown parameters are decrease as the sample size increase.

\section{Acknowledgements}

All praises and gratitude to ALLAH for giving me ability, knowledge and patience to complete this work. I wish to express my deepest gratitude to Professor. Ahmed. F. Attia and Dr. Salwa Mahmoud Samy Assar for their kind supervisions, creative ideas, fantastic advices, helpful suggestions, valuable discussions and comments, continuous encouragement and over all their valuable time and great experiences during the accomplishment of this paper. I want to express my deepest gratitude to my parents and my brothers for their continuous encouragement, great support, good help during the preparing of this paper. I wish to express my deepest gratitude to my future husband wafik wagih for his creative ideas, valuable advices and great help during preparing of this paper. Without his encouragement, this work would have never been complete.

\section{References}

[1] A. A. Abdel-Ghaly, A. F. Attia and H. M. Aly, "Estimation of The Parameters of Pareto Distribution and The Reliability Function Using Accelerated Life Testing with Censoring", Communications in Statistics Simulation and Computation, 27, (1998), 469-484.

[2] D. K. Al-MAutrairi, M. E. Gitany and D. Kundu, "A New Bivariate Distribution with Weighted Exponential Marginals and its Multivariate Generalization", Statistical Papers, 52 (4), (2011), 921-936.

[3] S. K. Ashour, A. A. Essam and H. Z. Muhammed, "Moment Generating Function of The Bivariate Generalized Exponential Distribution", Applied Mathematical Sciences, 3(59), (2009), 2911 - 2918.

[4] S. K. Ashour, A. A. Essam and H. Z. Muhammed, "Maximum Likelihood Estimation for The Baivariate Generalized Exponential Distribution Parameters using Type I Censored Data", Journal of Applied Sciences Research, 8(4), (2012), 1893-1900.

[5] A. F. Attia, H. M. Aly and S. O. Bleed, "Estimating and Planning Accelerated Life Test using Constant Stress for Generalized Logistic Distribution under Type-I Censoring", ISRN Applied Mathematics, 203618, (2011), 15 pages.

[6] A. F. Attia, H. M. Aly and H. Z. Muhammed, "Estimation of the Bivariate Generalized Linear Failure Rate Distribution under Different Censoring Schemes", Proceedings of the $47^{\text {th }}$ Annual Conference in Statistics, Computer Science, and Operations Research, Institute of Statistical Studies and Research, Cairo University, (2013).

[7] E. A. Elsayed, "Reliability Engineering", Addison Wesley longman, Inc, (1996).

[8] D. D. Hanagel, "Some Inference Results in Bivariate Exponential Distribution Based on Censored Samples", Communications in Statistics Theory and Methods, 21(5), (1992), 1273-1295.

[9] P. Houggard, B. Harvald and N. V. Holm, "Measuring the Similarities between the Lifetimes of Adult Danish Twins Born Between 18811930", Journal of Statistical Society Association, 87, (1992), 17-24

[10] D. Kundu and R. D. Gupta, "Bivariate Generalized Exponential Distribution", Journal of Multivariate Analysis, 100 (4), (2009), 581 - 593.

[11] D. Kundu and R. D. Gupta, "Absolute Continuous Bivariate Generalized Exponential Distribution", Journal of Advances in Statistical Analysis, 95 (2), (2011), 169 - 185.

[12] D. Kundu and A. K. Gupta, "On Bivariate Weibull-Geometric Distribution", Journal of Multivariate Analysis, 123, (2014), 19-29.

[13] J. F. Lawless, "Confidence Interval Estimation in the Inverse Power Law Model", Journal of the Royal Statistical Society: Series C, 25(2), (1976), 128-138.

[14] D. Y. Lin, W. Sun and Z. Ying, "Nonparametric Estimation of the Gap Time Distribution for Serial Events with Censored Data", Biometrikia, 86, (1999), 59-70.

[15] A. W. Marshall and I. Olikn, "A Multivariate Exponential Distribution", Journal of the American Statistical Association, 62, (1967), 30-44.

[16] W. Nelson, "Accelerated Testing: Statistical Models, Test Plans, and Data Analyses", New Yourk, John Wiley \& Sons, (1990).

[17] S. Shoaee and E. Khorram, "A New Absolute Continuous Bivariate Generalized Exponential Distribution", Statistical Planning and Inference, 142(7), (2012), 2203-2220.

[18] N. D. Singpurwalla, "A Problem in Accelerated Life Testing", Journal of American Statistical Association, 66, (2012), 841-845. 